\title{
Bernard Pouderon, (dir.), Histoire de la littérature grecque chrétienne
}

Introduction de Enrico Norelli et Bernard Pouderon. Paris, Éditions du Cerf, coll. «Initiation aux Pères de l'Église », 2008, 333 p.

Isabelle Jonveaux

\section{CpenEdition}

Journals

Édition électronique

URL : http://journals.openedition.org/assr/19803

DOI : $10.4000 /$ assr. 19803

ISSN : $1777-5825$

Éditeur

Éditions de l'EHESS

Édition imprimée

Date de publication : 1 octobre 2008

Pagination : 163-274

ISBN : 978-2-7132-2192-7

ISSN : 0335-5985

Référence électronique

Isabelle Jonveaux, « Bernard Pouderon, (dir.), Histoire de la littérature grecque chrétienne », Archives de sciences sociales des religions [En ligne], 144 | octobre-décembre 2008, document 144-52, mis en ligne le 04 février 2009, consulté le 21 septembre 2020. URL : http://journals.openedition.org/assr/19803 ; DOI : https://doi.org/10.4000/assr.19803

Ce document a été généré automatiquement le 21 septembre 2020

(c) Archives de sciences sociales des religions 


\section{Bernard Pouderon, (dir.), Histoire de la littérature grecque chrétienne}

Introduction de Enrico Norelli et Bernard Pouderon. Paris, Éditions du Cerf, coll. « Initiation aux Pères de l'Église », 2008, 333 p.

Isabelle Jonveaux

1 Ces trois cent quinze pages d'une précision remarquable ne sont que l'ouvrage introductif à une vaste Histoire de la littérature grecque chrétienne. Le but de cette introduction est d'une part, comme il se doit, de circonscrire le sujet, c'est pourquoi les préliminaires méthodologiques tentent d'abord de définir la littérature, la définition retenue étant celle de "la communication par des textes écrits », et d'autre part, de poser le cadre concret et historique dans lequel se développe cette littérature. D’où la question de la transmission de ces textes, des supports, mais aussi les formes employées, et même de l'histoire de l'étude de cette littérature.

2 Une fois retenue la définition de la littérature, intervient la question de savoir quelle réalité recouvre la littérature chrétienne, car certaines œuvres païennes peuvent s'en rapprocher. Le critère retenu est celui de la référence à Jésus Christ, « les écrits qui adoptent Jésus et son enseignement comme référence sont des écrits chrétiens et doivent figurer dans une histoire de la littérature chrétienne. " (Enrico Norelli p. 35). Cependant, l'Église est restée longtemps méfiante vis-à-vis d'une littérature critique du Nouveau Testament qu'elle ne pouvait pas contrôler car il y a bien quelque chose de la définition de la religion chrétienne qui va passer par cette littérature sans que ces textes émanent tous directement de l'institution ecclésiale en elle-même.

Le troisième chapitre, de Gilles Dorval sur les formes et modèles littéraires, et le cinquième, de Marie-Ange Calvet-Sebasti sur l'esthétique, abordent la question de la spécificité de la littérature chrétienne. Autrement dit, la littérature grecque chrétienne a-t-elle créé sa propre esthétique, sa propre forme littéraire ? Deux éléments premiers sont à considérer : d'une part, l'héritage hellénistique de ces auteurs et leur formation en rhétorique, et d'autre part, leur volonté de se recentrer uniquement sur le contenu, le message de foi en proclamant leur inculture et leur incapacité à écrire. L'idéal de simplicité se veut opposé à la rhétorique païenne, cependant les formes utilisées ne 
sont pas originales: extraits prophétiques, homélies, commentaires, poésie, genre épistolaire, etc. L'innovation de cette littérature tient beaucoup plus au message qu'elle véhicule qu'à sa forme. Et même si certains textes témoignent d'une réelle préoccupation esthétique (malgré sa négation), ils n'ont pas été reçus par le public comme des faits littéraires. La théologie qui se construit proclame qu'elle n'a pas besoin de beauté littéraire principalement pour se démarquer de la tradition de l'hellénisme. Nous pouvons alors nous demander si, finalement, ce n'est pas là que naît un certain type de littérature que nous disons aujourd'hui scientifique, qui s'attache au contenu et non à la forme.

4 Ces écrits ne sont pas étrangers à l'élaboration dogmatique de l'Église chrétienne, ils y ont participé activement. En effet, les travaux théologiques, notamment de christologie, ont servi de base aux premiers conciles. Par exemple, la question de la désignation du Christ et de la Trinité s'est élaborée petit à petit dans ces textes, ensuite ratifiés par les conciles. Marie-Anne Vannier souligne avec justesse qu'« en l'espace de quelques siècles, il y a eu plus d'évolution dogmatique que dans le millénaire suivant ", notamment en posant les bases du vocabulaire théologique.

5 Les chapitres de Rémi Gounelle et Martin Wallraff abordent enfin la question du support, et de la transmission de ces écrits. Il serait illusoire de croire que ces textes des premiers siècles sont parvenus à l'identique jusqu'à nos jours. Les problèmes de rééditions «adaptées », inversions de pages, lacunes comblées par d'autres manuscrits, incendie, ou de non copiage ou non réédition tout simplement font que les ouvrages que nous possédons aujourd'hui ne sont qu'une partie d'un ensemble plus vaste et parfois plus ou moins proches du texte original. À cela s'ajoutent des travaux explicites de falsification, notamment à la bibliothèque d'Alexandrie dans le cadre de la propagande monophysite. Au cours des siècles, de nombreuses œuvres de regroupement des textes furent tentées, l'œuvre immense des bénédictins de SaintMaur est à retenir, ou encore la collection de Migne (1800-1875) Patrologiae Cursus Completus, malheureusement non achevée du fait d'un incendie dans son imprimerie. Selon M. Wallraff, le travail de Migne "est le transfert de la "révolution industrielle" dans le domaine de la patristique » (p. 247) car son but était d'élever le niveau de la culture catholique par une entreprise tournée vers le profit économique. Ce travail de rassemblement et de réédition des textes patristiques est l'œuvre aujourd'hui de la collection « Sources Chrétiennes » notamment, qui tente de surcroît de redécouvrir des textes non imprimés jusqu'alors. L'outil informatique peut aussi se trouver d'une grande utilité aujourd'hui, principalement pour des textes excessivement longs comme les commentaires des Psaumes par saint Augustin par exemple, dont l'utilisation sera beaucoup plus simple en consultation par Internet qu'en lecture linéaire.

6 Le dernier chapitre de l'ouvrage enfin, par Benoît Gain est une recension d'instruments de travail pour l'étude de la littérature grecque chrétienne ancienne qui seront sans doute d'une grande utilité à ceux qui s'y intéressent. Revues, ouvrages classés thématiquement, outils informatiques et bibliothèques se trouvent ici réunis pour faciliter l'approche de ce sujet.

7 Finalement, cet ouvrage est un excellent outil pour l'étude de cette littérature, néanmoins il pourra sembler d'un abord difficile dans sa précision à ceux qui ne sont pas familiers de ce sujet. Il propose de manière synthétique mais dense un panorama des diverses questions qui se posent au sujet de cette littérature, citant les principaux auteurs et leurs écrits, inscrits dans le contexte historique, religieux et littéraire. On 
attend donc avec impatience la suite de cette collection qui devrait comprendre au total six volumes, des écrits de Paul au concile de Chalcédoine (465). À suivre. 